\title{
Neutron Star Kicks Affected by Standing Accretion Shock Instability for Core-Collapse Supernovae
}

\author{
Wakana Iwakami Nakano ${ }^{1}$, Kei Kotake ${ }^{2}$, Naofumi Ohnishi ${ }^{3}$, \\ Shoichi Yamada ${ }^{4}$, and Keisuke Sawada ${ }^{3}$ \\ ${ }^{1}$ Institute of Fluid Science, Tohoku University, 2-1-1 Katahira, Aoba-ku, Sendai, Miyagi, \\ 980-8577, Japan \\ email: wakana@dragon.ifs.tohoku.ac.jp \\ ${ }^{2}$ Division of Theoretical Astronomy/Center for Computational Astrophysics, National \\ Astronomical Observatory of Japan, 2-21-1, Osawa, Mitaka, Tokyo, 181-8588, Japan \\ ${ }^{3}$ Department of Aerospace Engineering, Tohoku University, 6-6-01 Aramaki-Aza-Aoba, \\ Aoba-ku, Sendai, 980-8579, Japan \\ ${ }^{4}$ Advanced Research Institute for Science and Engineering, Waseda University, 3-4-1 Okubo, \\ Shinjuku, Tokyo, 169-8555, Japan
}

\begin{abstract}
We investigate a proto-neutron star kick velocity estimated from kinetic momentum of a flow around the proto-neutron star after the standing accretion shock instability grows. In this study, ten different types of random perturbations are imposed on the initial flow for each neutrino luminosity. We found that the kick velocities of proto-neutron star are widely distributed from $40 \mathrm{~km} \mathrm{~s}^{-1}$ to $180 \mathrm{~km} \mathrm{~s}^{-1}$ when the shock wave reaches $2000 \mathrm{~km}$ away from the center of the star. The average value of kick velocity is $115 \mathrm{~km} \mathrm{~s}^{-1}$, whose value is smaller than the observational ones. The kick velocities do not depend on the neutrino luminosity.
\end{abstract}

Keywords. supernovae, shock waves, hydrodynamics, instabilities.

\section{Introduction}

Pulsars are highly rotating and magnetized compact objects with periodic electromagnetic radiations. The velocities of their proper motions tend to be larger than those of other type of astronomical objects. The mean speeds of them are about $400 \mathrm{~km} \mathrm{~s}^{-1}$, and the maximum velocity is around $1600 \mathrm{~km} \mathrm{~s}^{-1}$ (Hobbs (2005)). The mechanism of pulsar kicks has not been understood clearly yet. Pulsars are considered as neutron stars. Neutron stars are produced by core-collapse supernova explosions of massive stars. Some core-collapse supernova remnants have shown that non-spherically symmetric explosions occur (Winkler \& Petre (2007)). Surrounding matters having nonuniformly distributed kinetic momenta might give a large kick velocity for a neutron star. Standing accretion shock instability (SASI) is regarded as playing a key role to the asymmetric explosions of core-collapse supernovae. The SASI makes the spherical shock wave deformed, and high entropy bubbles are generated behind the shock wave. The behavior of fluids inside and outside the bubbles produces the non-spherically symmetric distribution of kinetic momenta around the proto-neutron star (PNS). The kick velocity of PNS have been investigated with axisymmetric two-dimensional (2D) (Sheck (2006), Nordhaus (2010)) and non-axisymmetric three-dimensional (3D) (Wongwathanarat (2010)) simulations for core-collapse supernovae in recent years. These studies have obtained hundreds $\mathrm{km} \mathrm{s}^{-1}$ of kick velocities. 

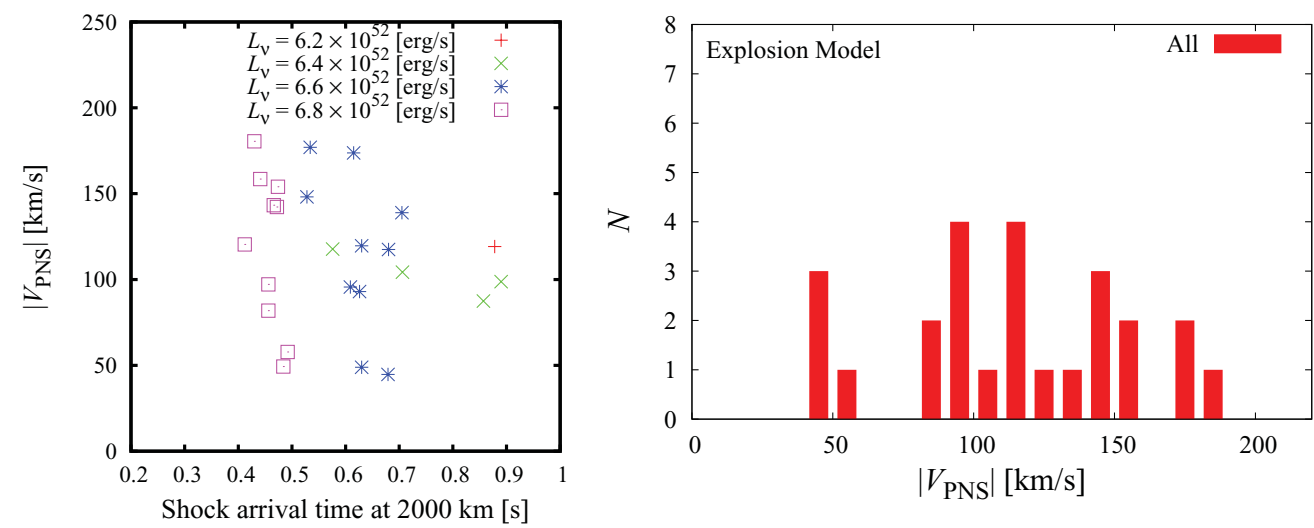

Figure 1. The kick velocities classified into neutrino luminosities as a function of shock arrival time at $2000 \mathrm{~km}$ (left panel) and the kick velocity distribution (right panel).

Our objective is to calculate the kick velocities from the results of three-dimensional simulations for core-collapse supernovae. We impose ten different types of random perturbations for each neutrino luminosity in the range $5.8-6.8 \times 10^{52} \mathrm{erg} \mathrm{s}^{-1}$. The computational procedure is the same as explained in our previous paper (Iwakami (2008)).

\section{Results and Discussions}

We estimate the kick velocities from the equations described as

$$
v_{\mathrm{PNS}}(t)=-\mathbf{P}_{\mathrm{gas}}(t) / M_{\mathrm{PNS}}(t) \quad \mathbf{P}_{\mathrm{gas}}(t)=\int_{R_{\mathrm{PNS}}<r<\infty} \rho \mathbf{v} d V .
$$

Fig. 1 shows in the left panel the shock arrival time dependence of the kick velocities. The shock arrival time is defined as the time when the shock wave reaches $2000 \mathrm{~km}$. The kick velocity does not depend on the neutrino luminosity. The higher neutrino luminosity, the shorter the arrival time tends to be. So the kick velocity does not correlate with the speed of shock propagation.

Fig. 1 displays in the right panel the kick velocity distribution for 25 explosion models. The kick velocity is distributed from $40 \mathrm{~km} \mathrm{~s}^{-1}$ to $180 \mathrm{~km} \mathrm{~s}^{-1}$ Average value is $115 \mathrm{~km}$ $\mathrm{s}^{-1}$ which is smaller than the value of $400 \mathrm{~km} \mathrm{~s}^{-1}$ obtained by observation. The velocities are measured when the shock wave reaches $2000 \mathrm{~km}$. If we use a larger computational region than $2000 \mathrm{~km}$ and calculate for longer time, the kick velocities would be larger.

\section{Conclusions}

The resulting kick velocities, distributed from $90 \mathrm{~km} \mathrm{~s}^{-1}$ to $180 \mathrm{~km} \mathrm{~s}^{-1}$ do not depend on the arrival time of the shock wave at $2000 \mathrm{~km}$ from the center of the star.

\section{References}

Hobbs, G., Lorimer, D. R., Lyne, A. G., \& Kramer M. 2005, MNRAS, 360, 974

Winkler, P. F. \& Petre, R. 2007, ApJ, 670, 635

Scheck, L., Kifonidis, K., Janka, H.-Th., \& M'uller, E. 2006, A\& A, 457, 963

Nordhaus, J., Brandt, T. D., Burrows, A., Livne, E., \& Ott, C. D. 2010, Phys. Rev. D, 82, 103016

Wongwathanarat, A., Janka, H.-Th., \& M'uller, E. 2010, ApJ, 725, L106

Iwakami, W., Kotake, K., Ohnishi, N., Yamada, S., \& Sawada, K. 2008, ApJ, 678, 1207 